\section{A prescrição semanal de sulfato ferroso pode ser altamente efetiva para reduzir níveis endêmicos de anemia na infância}

\section{Long-term preventive mass prescription of weekly doses of iron sulfate may be highly effective to reduce endemic child anemia}

\author{
Carlos Augusto Monteiro \\ Departamento de Nutrição \\ Núcleo de Pesquisas Epidemiológicas em Nutrição e Saúde - NUPENS/USP \\ Faculdade de Saúde Pública \\ Universidade de São Paulo \\ Av. Dr. Arnaldo, 715 \\ 01246-904 São Paulo- SP, Brasil \\ carlosam@usp.br
}

\section{Sophia Cornbluth Szarfarc}

Departamento de Nutrição

Núcleo de Pesquisas Epidemiológicas em Nutrição e Saúde - NUPENS/USP

Faculdade de Saúde Pública

Universidade de São Paulo

Gisela Soares Brunken

Departamento de Ciência e Tecnologia de Alimentos

Faculdade de Enfermagem e Nutrição

Universidade Federal de Mato-Grosso

\section{Rainer Gross}

Gesellschaft für Technische Zusammenarbeit (GTZ Gmbh)

Eschborn, Germany

\section{Wolney Lisboa Conde}

Departamento de Nutrição e

Núcleo de Pesquisas Epidemiológicas em Nutrição e Saúde - NUPENS/USP

Faculdade de Saúde Pública

Universidade de São Paulo

\section{Auxílio financeiro}

Fundação de Amparo à Pesquisa do Estado de São Paulo - FAPESP (Processo no: 94/3493-5) e Ministério da Saúde (Convênio MS/FUSP n: 28/96).

A versão inglesa do presente artigo foi publicada pelo Boletim de Alimentação e Nutrição da Universidade das Nações Unidas (Food Nutr Bull 22 (1): 53-61; 2001).

\section{Resumo}

A anemia por deficiência de ferro em crianças é um dos maiores problemas nutricionais enfrentados pelos países em desenvolvimento. Estudos controlados indicam que doses intermitentes de sais de ferro podem ter eficácia semelhante à obtida com o esquema tradicional diário. O objetivo desse estudo é avaliar, em uma população onde a anemia na infância é endêmica, a efetividade da prescrição preventiva de doses semanais de sulfato ferroso a todas as crianças entre seis e 59 meses de idade por um período de seis meses.

Crianças dos grupos controle e intervenção foram selecionadas a partir de uma amostra aleatória da população de crianças da cidade de São Paulo. Os pais das crianças do grupo intervenção receberam orientação nutricional e frascos de solução de sulfato ferroso com a instrução de ofertá-la aos filhos uma vez por semana até a próxima visita da equipe de pesquisa, que ocorreria em aproximadamente seis meses. Os pais das crianças do grupo controle receberam somente orientação nutricional. O efeito da intervenção foi avaliado por mudanças na concentração da hemoglobina e na prevalência de anemia. As comparações entre os dois grupos foram baseadas na "intençãode-tratar" e todas as estimativas foram ajustadas para concentração inicial de hemoglobina, idade inicial, duração do seguimento e renda familiar.

O ganho médio de hemoglobina devido à intervenção foi de $4,0 \mathrm{~g} / \mathrm{l}$ e a queda na prevalência de anemia foi de mais de $50 \%$. A intervenção foi particularmente eficiente em prevenir o declínio da concentração de hemoglobina durante os dois primeiros anos de vida. Esse estudo demonstra que, em condições similares àquelas que poderiam facilmente ser reproduzidas por programas regulares de saúde pública, a prescrição universal de doses semanais de sulfato ferroso reduz significativamente o risco de anemia na infância.

Palavras-chave: Anemia ferropriva. Suplementação semanal de ferro. Suplementos dietéticos. Criança. Intervenção nutricional. 


\section{Abstract}

Iron-deficiency anemia in children remains one of the most important nutritional problems faced by developing countries. Wellcontrolled efficacy studies show that intermittent iron supplementation can improve children's iron status as well as the traditional daily schedule. This gives new impetus to controlling child anemia by weekly preventive iron supplementation. The objective of this study is to evaluate the effectiveness of long-term preventive provision of weekly doses of iron sulfate to all children from 6 to 59 months of age, in a child population in which anemia is highly prevalent.

Children from both the intervention and control groups were selected from a random crosssectional sample of the child population of the city of São Paulo, Brazil. Parents in the intervention group were visited in their homes and received nutrition education plus a solution of iron sulfate with the request to give it to their children once a week until a follow-up visit occurred in approximately six months. Parents in the control group received only nutrition education. The effect of the intervention was assessed by changes in hemoglobin concentration and the prevalence of anemia. Comparisons between the two groups were made based on an intention-to-treat approach, and all estimates were adjusted for initial hemoglobin concentration, initial age, total duration of follow-up and family income.

The average hemoglobin gain due to the intervention was $4.0 \mathrm{~g} / \mathrm{l}$, with a fall of more than $50 \%$ in the prevalence of anemia among the children. The intervention was particularly effective in preventing declines in hemoglobin concentration during the first two years of life. This study demonstrates that long-term preventive weekly iron supplementation for preschool children significantly reduces the risk of anemia under conditions similar to those possible in routine public health programs.

Keywords: Anemia. Iron-deficiency. Iron weekly supplementation. Dietary supplements. Children. Nutritional intervention.

\section{Introdução}

A anemia por deficiência de ferro durante a idade pré-escolar permanece como um dos mais importantes problemas nutricionais encontrados nos países em desenvolvimento. Estima-se que, nesses países, uma em cada duas crianças menores de cinco anos de idade seja anêmica (concentração de hemoglobina menor que $110 \mathrm{~g} / \mathrm{l})^{1}$. Ingestão deficiente de ferro biodisponível, elevado requerimento do mineral associado com o crescimento e infestações parasitárias parecem ser os principais fatores responsáveis pela elevada prevalência de anemia infantil nos países em desenvolvimento ${ }^{2}$. As conseqüências da deficiência de ferro nos primeiros anos de vida são extremamente relevantes, incluindo prejuízo no desenvolvimento cognitivo e baixo rendimento acadêmico na idade escolar ${ }^{3-5}$. Diferentemente do observado com relação às deficiências de vitamina $\mathrm{A}$, iodo, proteína e energia, não há relato de progresso significativo no controle dos níveis endêmicos de anemia ferropriva existentes na população infantil de países em desenvolvimento ${ }^{6}$.

Inquérito domiciliar realizado em meados da década de 90 na cidade de São Paulo evidenciou que cerca de metade da população entre 6 e 59 meses de idade encontravase anêmica, sendo de quase $70 \%$ a prevalência de anemia entre os 6 e os 23 meses de idade. Dados coletados pelo mesmo inquérito sugerem que a deficiência de ferro na dieta é o principal determinante da alta prevalência de anemia infantil em São Paulo: $76,7 \%$ das crianças estudadas ingeriam diariamente menos do que $10 \mathrm{mg}$ de ferro enquanto apenas cerca de $10 \%$ apresentavam-se infectadas por parasitas intestinais, sendo que nenhuma por ancilostomídeos?

Fortificação de alimentos com ferro e distribuição de suplementos medicamentosos com sais de ferro pela rede pública de saúde são as duas intervenções com maior potencial para controlar a anemia ferropriva em crianças pequenas ${ }^{8}$. Porém, em países em desenvolvimento, alimentos fortificados com ferro são usualmente muito caros e, 
portanto, pouco disponíveis para a maioria da população, enquanto a distribuição de suplementos medicamentosos em serviços de saúde tem baixa cobertura e está usualmente restrita a mulheres gestantes ${ }^{8}$. Estudos recentes, bem controlados, em crianças pré-escolares asiáticas com deficiência de ferro, demonstraram que a suplementação medicamentosa com sais de ferro, supervisionada, utilizando-se esquema de dosagem intermitente (uma ou duas vezes por semana), produziu impacto em indicadores do estado nutricional relativo a ferro semelhante ao obtido com a suplementação diária ${ }^{9-11}$. Essas demonstrações deram um novo ímpeto para a estratégia de controle da anemia em crianças por meio da distribuição de suplementos de ferro. A proposta do presente estudo é avaliar se, em uma população infantil onde a anemia é endêmica e a ingestão de ferro é claramente deficiente, uma intervenção baseada na simples prescrição universal de doses semanais de sulfato ferroso para crianças de 6 a 59 meses de idade pode melhorar a concentração de hemoglobina e reduzir a prevalência da anemia na infância.

\section{Métodos}

\section{População de estudo e desenho de investigação}

O presente estudo foi aninhado em um amplo inquérito transversal sobre condições de saúde e nutrição na infância realizado na cidade de São Paulo entre setembro de 1995 e setembro de $19966^{7}$. O referido inquérito visitou ao longo do período uma amostra representativa dos domicílios da cidade ( $\mathrm{n}=4.560)$ e dosou, nos próprios domicílios, empregando hemoglobinômetros portáteis, a concentração de hemoglobina de todas as crianças com idades entre 6 e 59 meses $(\mathrm{n}=1.158)$. Distritos com baixa, média e alta renda familiar foram aleatoriamente percorridos ao longo do período de realização da pesquisa e não foram constatadas variações sazonais na concentração de hemoglobina das crianças. Os pais de todas as crianças examinadas consentiram na participação, e o estudo foi aprovado pelo Comitê de Ética da Faculdade de Saúde Pública da Universidade de São Paulo.

O conjunto das crianças examinadas nas visitas domiciliares realizadas no período de setembro de 1995 a março de 1996 ( $n=635)$ constituiu o grupo controle do estudo. Os pais dessas crianças foram informados sobre o resultado do exame da concentração de hemoglobina e encorajados, independentemente da presença ou ausência de anemia, a melhorar o aporte da alimentação infantil em alimentos ricos em ferro, como carnes, feijões e verduras de folhas verdesescuras. Em caso de presença de anemia, obedecendo a princípios éticos, os pais das crianças do grupo controle foram aconselhados a procurar tratamento no serviço de saúde que costumavam utilizar.

O conjunto das crianças examinadas de abril a setembro de 1996 ( $n=499$ ) constituiu o grupo intervenção do estudo. Os pais dessas crianças, além de também serem informados sobre o resultado do exame da concentração de hemoglobina e de serem encorajados a aumentar o aporte de ferro na dieta infantil, receberam, independentemente da presença ou ausência de anemia, frascos de um xarope de sulfato ferroso com instruções para oferecer o produto a seus filhos uma vez por semana, continuamente, até o retorno da equipe da pesquisa, que deveria ocorrer em cerca de seis meses. Aos pais das crianças do grupo intervenção foi também entregue um calendário especialmente confeccionado pelo estudo para registro dos dias em que a criança efetivamente ingerisse o xarope de sulfato ferroso.

Após um período de cerca de 6 a 7 meses, as crianças dos grupos controle e intervenção foram revisitadas para nova avaliação de suas condições de saúde, incluindo nova dosagem da concentração de hemoglobina. Durante a segunda visita ao domicílio, os calendários deixados com as famílias do grupo intervenção foram recolhidos e, no caso de calendários sem registro $(42 \%$ dos casos), os pais das crianças responderam um questionário com perguntas sobre a freqüência com que o xarope de sulfato ferroso 
tinha sido efetivamente administrado à criança. Ainda durante a segunda visita, os pais das crianças do grupo intervenção foram perguntados sobre a ingestão de outros preparados com ferro, além do produto deixado pela equipe da pesquisa; e os pais das crianças do grupo controle foram indagados a respeito da eventual ingestão de qualquer preparado com ferro no período decorrido desde a visita inicial. Nenhum contato entre as famílias dos dois grupos de crianças e a equipe de pesquisa ocorreu no intervalo entre as duas visitas.

Devido a mudanças de endereço, a segunda avaliação da concentração de hemoglobina não pôde ser realizada em 76 (12,0\%) das 635 crianças do grupo controle e em 75 $(15,0 \%)$ das 499 crianças do grupo intervenção. Quase metade das crianças do grupo controle $(46,7 \%$, ou 261 das 559 crianças) recebeu suplementos com ferro durante o seguimento. Conforme esperado, a maioria das crianças do grupo controle que receberam suplementos de ferro eram crianças anêmicas cujos pais seguiram os conselhos da equipe de pesquisa e procuraram serviço de saúde para tratar a enfermidade. Cinqüenta e cinco das 424 crianças do grupo intervenção $(11,0 \%)$, divididas igualmente entre anêmicas e não anêmicas, receberam outro suplemento com ferro que não o ofertado pelo estudo. Esses dois grupos de crianças $(261+55)$ foram excluídos do estudo de avaliação do impacto da intervenção. Crianças cuja duração do seguimento ficou aquém de cinco meses ou excedeu 8,9 meses foram também excluídas da avaliação ( 5 crianças do grupo controle e 23 do grupo intervenção). Após essas exclusões, a média de duração do período de seguimento ficou em 6,9 $\pm 0,7$ meses para o grupo controle e $7,0 \pm 0,8$ meses para o grupo intervenção. Sendo assim, a eficiência da suplementação preventiva com ferro semanal descrita nesse estudo foi avaliada com base em 293 crianças controles e 346 crianças do grupo intervenção. É evidente que as perdas de seguimento e, especialmente, as exclusões podem ter distorcido a randomização esperada dos grupos controle e intervenção ao final do experimento, em particular com relação à concentração inicial de hemoglobina. Essa possibilidade será avaliada e levada em conta na análise estatística dos resultados obtidos pelo estudo.

\section{Xarope de ferro}

O xarope com sulfato ferroso ofertado às crianças do grupo intervenção foi preparado pelo Departamento de Nutrição da Faculdade de Saúde Pública da Universidade de São Paulo. Foi embalado em ampolas de vidro marrom, com $85 \mathrm{ml}$, contendo cada uma $20 \mathrm{mg} / \mathrm{ml}$ de ferro elementar. A quantidade total de ferro elementar em cada frasco foi calculada para ser bem menor do que a dose letal, em caso de a criança acidentalmente ingerir todo o seu conteúdo ${ }^{12}$. Cada frasco foi embalado com uma pequena colher transparente de plástico com marcas indicativas dos diferentes volumes da solução. A ingestão recomendada por semana variou conforme a idade da criança ao início da intervenção, de modo a prover $30 \mathrm{mg}$ de ferro elementar para crianças entre 6 e 9 meses, $40 \mathrm{mg}$ para crianças entre 10 e 17 meses, $50 \mathrm{mg}$ para crianças entre 18 e 29 meses, $60 \mathrm{mg}$ para crianças entre 30 e 35 meses, e $80 \mathrm{mg}$ para crianças entre 36 e 59 meses de idade. A ingestão recomendada correspondia a aproximadamente $4 \mathrm{mg}$ de ferro elementar por quilograma de peso. Os pais das crianças mais novas (menores de 30 meses) receberam um frasco de solução, enquanto os outros receberam dois frascos.

\section{Coleta de dados}

O mesmo procedimento de coleta de dados foi utilizado para os grupos controle e intervenção. Como parte do protocolo do estudo transversal, os pais das crianças foram entrevistados para determinar as características demográficas e socioeconômicas da família, incluindo a data de nascimento da criança, número total de residentes no domicílio e todas as fontes de renda familiar. Uma amostra de sangue capilar foi obtida nos momentos inicial e final do estudo, puncionando-se a polpa digital do dedo médio ou 
anular da criança. A concentração de hemoglobina era determinada no domicílio, imediatamente após a punção digital, pelo método da cianometahemoglobina empregado pelo fotômetro portátil (HemoCue ${ }^{\circledR}$, Angelholm, Sweden) ${ }^{13}$. Medidas de peso e altura foram coletadas para construir indicadores do estado nutricional geral das crianças. O peso foi aferido por balanças microeletrônicas (modelo 7307, Soehnle, Alemanha) com aproximação de 0,1 kg, estando as crianças despidas. Medidas de comprimento com aproximação de $0,1 \mathrm{~cm}$ foram tomadas em crianças com menos de dois anos de idade, utilizando-se antropômetros horizontais especialmente confeccionados para a pesquisa (AHRTAG, London). Em crianças a partir de dois anos de idade, aferiu-se a estatura com aproximação de $0,1 \mathrm{~cm}$, utilizando-se antropômetro vertical da marca SECA (SECA, bodymeter 208).

Amostras de fezes para exame parasitológico foram coletadas na visita inicial feita às crianças dos grupos controle e intervenção ${ }^{14}$. Os exames parasitológicos foram realizados ao longo do período, entre a primeira e a segunda visita no laboratório de parasitologia do Instituto de Ciências Biomédicas da Universidade de São Paulo, e os resultados foram informados aos pais das crianças durante a segunda visita. As crianças com exames positivos (em sua maioria casos de Giardia duodenalis e Ascaris lumbricoides, não havendo casos de ancilostomídeos) receberam da equipe de pesquisa tratamento medicamentoso apropriado. A ingestão diária aproximada de energia, proteína, vitamina A e ferro foi estimada no início do seguimento em uma subamostra sistemática das crianças dos grupos controle e intervenção, utilizando-se inquérito recordatório de 24 horas $^{15}$. A ingestão de alimentos foi transformada em nutrientes, utilizando-se o aplicativo "Virtual Nutri” ${ }^{16}$, e as porcentagens de recomendação dietética foram calculadas segundo as recomendações internacionais ${ }^{17,18}$.

\section{Análise estatística}

Diversos testes e análises foram empre- gados para avaliar o impacto resultante da intervenção ${ }^{19}$. Testes t para comparações de médias e testes baseados na distribuição do qui quadrado para comparação de proporções foram usados para contrastar as distribuições iniciais dos grupos controle e intervenção quanto a variáveis que poderiam influenciar mudanças na concentração de hemoglobina durante o estudo, a saber: concentração de hemoglobina, idade, renda familiar, parasitas intestinais, peso/altura, altura/idade, peso/idade, ingestão diária e porcentagem da recomendação de ingestão diária de energia, proteína, vitamina A e ferro. Testes $t$ foram empregados para comparar os dois grupos quanto à variação (incremento ou decréscimo) na concentração de hemoglobina entre o início e o final do seguimento. Análises de regressão linear foram utilizadas para estimar a variação na concentração de hemoglobina que se poderia atribuir à intervenção. Essa variação é dada pelo coeficiente de regressão de uma variável que indica o status controle ou intervenção (0 ou 1) de cada criança em um modelo linear que tem a variação da concentração de hemoglobina durante o seguimento como variável dependente. Análises de regressão logística foram realizadas para modelar os riscos relativos (razão de chances) de aumento ou decréscimo relevante na concentração de hemoglobina das crianças do grupo intervenção quando comparadas às crianças do grupo controle e também para modelar a probabilidade de anemia em cada um dos grupos ao final do seguimento. As análises de regressão linear e logística forneceram estimativas brutas do efeito da intervenção e estimativas ajustadas segundo valores iniciais de potenciais variáveis de confusão. Foram consideradas potenciais variáveis de confusão todas as variáveis cujas distribuições iniciais fossem diferentes entre os grupos controle e intervenção ( $\mathrm{p}<$ 0,20 ), e mais a duração total do seguimento (tempo decorrido entre as duas visitas). A faixa de idade da criança no início do seguimento e o nível de adesão da mesma à intervenção (estimado a partir das marcações no calendário ou por informações prestadas 
pelos pais) foram investigados como possíveis modificadores do impacto da intervenção. Todas as análises estatísticas foram realizadas utilizando-se o aplicativo "Stata" ${ }^{20}$. O aplicativo "EpiInfo" $6^{21}$ foi usado para entrada dos dados e para gerar indicadores antropométricos baseados nos padrões do "National Center for Health Statistics / World Health Organization" ${ }^{22}$.

\section{Resultados}

A Tabela 1 apresenta características relevantes no início do estudo referentes às crianças que constituíram os grupos controle e intervenção considerados neste estudo. Conforme o esperado, devido à elevada exclusão dos indivíduos anêmicos, as crianças do grupo controle apresentam concentração média de hemoglobina superior (114,2 contra 111,4 g/l) e prevalência de anemia inferior $(30,1 \%$ contra $41,7 \%)$ com rela- ção às crianças do grupo intervenção. Provavelmente pelo mesmo motivo, as crianças do grupo controle têm médias de idade e de renda familiar ligeiramente maiores do que as crianças do grupo intervenção, uma vez que a prevalência de anemia está inversamente relacionada à idade e à renda. Notese que as diferenças entre os grupos controle e intervenção desaparecem ou são substancialmente reduzidas quando se considera o conjunto das crianças originalmente alocadas para cada um dos grupos (dados não apresentados).

Outras características das crianças que poderiam influenciar o comportamento da concentração de hemoglobina ao longo do seguimento, como o estado nutricional geral da criança, medido por indicadores antropométricos, a presença de parasitas intestinais e os níveis de ingestão de energia, proteína, ferro e vitamina A, apresentaram distribuições basais semelhantes em ambos

Tabela 1 - Características das crianças de 6 a 59 meses de idade dos grupos controle e intervenção no início do estudo*.

Table 1 - Baseline characteristics of 6- to 59-month-old children from the control and intervention groups*.

\begin{tabular}{lccc}
\hline Características das crianças & Grupo Controle & Grupo Intervenção & $\mathrm{p}$ \\
\hline Demográficas e antropométricas & $\mathrm{n}=293$ & $\mathrm{n}=346$ & \\
Idade (meses) & $36,4 \pm 0,9$ & $34,1 \pm 0,9$ & 0,07 \\
Renda familiar mensal per capita (R\$/m) & $298,6 \pm 20,5$ & $260,1 \pm 19,0$ & 0,17 \\
Concentração de hemoglobina (g/l) & $114,2 \pm 0,9$ & $111,4 \pm 0,9$ & 0,04 \\
Anemia (\%) & 30,1 & 41,7 & $<0,01$ \\
Peso/ldade (NCHS z-score) & $-0,03 \pm 0,07$ & $-0,08 \pm 0,05$ & 0,56 \\
Altura/Idade (NCHS z-score) & $0,00 \pm 0,06$ & $-0,09 \pm 0,05$ & 0,28 \\
Peso/Altura (NCHS z-score) & $0,08 \pm 0,06$ & $0,09 \pm 0,05$ & 0,91 \\
Parasitas Intestinais (\%) & 12,7 & 11,3 & 0,61 \\
\hline Ingestão alimentar diária & $\mathrm{n}=136$ & $\mathrm{n}=166$ & \\
Energia (kcal / dia) & $1.405,8 \pm 58,9$ & $1,410,3 \pm 51,0$ & 0,95 \\
Energia (\% RDA) & $103,9 \pm 4,4$ & $109,0 \pm 4,2$ & 0,39 \\
Proteína (g /dia) & $54,5 \pm 2,5$ & $52,6 \pm 2,4$ & 0,59 \\
Proteína (\% RDA) & $343,9 \pm 15,8$ & $346,3 \pm 15,4$ & 0,91 \\
Vitamina A ( $\mu$ ER ER / dia) & $763,4 \pm 128,6$ & $865,8 \pm 138,9$ & 0,59 \\
Vitamina A (\% RDA) & $192,8 \pm 32,2$ & $219,7 \pm 34,8$ & 0,57 \\
Ferro (mg / dia) & $7,7 \pm 0,4$ & $7,6 \pm 0,4$ & 0,90 \\
Ferro (\% RDA) & $77,0 \pm 4,0$ & $76,0 \pm 4,0$ & 0,90 \\
\hline
\end{tabular}

Valores com \pm referem-se a média \pm erro padrão.

$\mathrm{NCHS}=$ National Center for Health Statistics; RDA = Recommended Dietary Allowance; $\mathrm{ER}=$ equivalentes de retinol. Plus-minus values are means \pm SE. National Center for Health Statistics Z scores: WAZ, weight-for-age; HAZ height-for-age; $\mathrm{WHZ}$, weight-for-height; $\mathrm{RDA}$, recommended dietary allowance; $\mathrm{RE}$, retinol equivalent. 
os grupos. É interessante notar que a distribuição dos indicadores antropométricos considerados mostra superposição com o padrão de referência utilizado, em ambos os grupos de crianças, o que indica que o estado nutricional geral da população estudada é satisfatório. Indicação semelhante advém dos dados da subamostra referentes à ingestão diária de energia, proteína e retinol, mas não para o nutriente ferro, que se mostra claramente deficiente na população. Nos dois grupos estudados, poucas crianças estavam sendo amamentadas e quase todas consumiam leite de vaca, sendo cerca de 500 ml o consumo médio diário desse alimento nos dois grupos (dados não apresentados).

A Tabela 2 descreve a variação observada na concentração de hemoglobina entre a primeira e a segunda visita. Embora se registre aumento na concentração de hemoglobina nos dois grupos de crianças, nota-se que o aumento médio é duas vezes maior no grupo intervenção: 10,0 g/l contra $4,5 \mathrm{~g} /$ 1 no grupo controle. A vantagem média do grupo intervenção em relação ao grupo controle reduz-se de 5,5 g/l para 4,0 g/l quando ajustada pela análise de regressão linear múltipla para as diferenças iniciais que separam os dois grupos quanto à concentra- ção inicial da hemoglobina, à idade ao início do seguimento (6-11, 12-17, 24-35, 36-47 e 48-59 meses de idade), à renda familiar per capita (aferida durante a primeira visita aos domicílios) e à duração total do seguimento. Tanto a vantagem bruta quanto a vantagem ajustada do grupo intervenção foram estatisticamente significativas ( $p<0,01$ ). Ressalte-se que todas as potenciais variáveis de confusão incluídas no modelo linear alcançaram significância estatística ( $\mathrm{p}<0,05$, dados não apresentados).

A Tabela 3 mostra a distribuição das crianças estudadas de acordo com possíveis modificadores do impacto da intervenção: a idade no início do seguimento e o grau de adesão à intervenção. Um alto nível de adesão (equivalente à ingestão efetiva do suplemento em pelo menos $75 \%$ das semanas decorridas entre a primeira e a segunda visita ao domicílio) foi encontrado em $62 \%$ de todas as crianças para as quais foi fornecido o suplemento ( $54 \%$ para as crianças entre 6 e 17 meses de idade e $64 \%$ para as crianças mais velhas).

A Tabela 4 provê estimativas do ganho médio na concentração de hemoglobina devido à intervenção. Essas estimativas, estratificadas de acordo com idade da cri-

Tabela 2 - Variações médias na concentração de hemoglobina ( $\mathrm{Hb}$ em g/l) em crianças de 6 a 59 meses de idade ao longo de 5 a 8 meses de seguimento observadas nos grupos controle e intervenção.

Table 2 - Average changes in hemoglobin ( $\mathrm{Hb}$ ) concentration ( $\mathrm{g} / \mathrm{l})$ in 60- to 59-month-old children over the 5 to 8-month followup in control and intervention groups.

\begin{tabular}{|c|c|c|c|c|c|c|c|c|c|c|c|}
\hline \multirow[t]{3}{*}{ Grupo } & \multirow[t]{3}{*}{$\mathrm{n}$} & \multirow{2}{*}{\multicolumn{2}{|c|}{ Hb no início }} & \multirow{2}{*}{\multicolumn{2}{|c|}{ Hb no final }} & \multirow{2}{*}{\multicolumn{2}{|c|}{ Incremento de $\mathrm{Hb}$}} & \multicolumn{4}{|c|}{$\begin{array}{l}\text { Ganho líquido de } \mathrm{Hb} \\
\text { devido à intervenção }\end{array}$} \\
\hline & & & & & & & & \multicolumn{2}{|c|}{ Bruto } & \multicolumn{2}{|c|}{ Ajustado* } \\
\hline & & & (EP) & & (EP) & & (EP) & & $(\mathrm{EP})$ & & (EP) \\
\hline Controle & 293 & 114,2 & $(1,0)$ & 118,7 & $(0,8)$ & 4,5 & $(1,0)$ & - & - & - & - \\
\hline Intervenção & 346 & 111,4 & $(0,9)$ & 121,4 & $(0,7)$ & $10,0^{+}$ & $(0,8)$ & $5,5^{\S}$ & $(1,3)$ & $4,0^{\S}$ & $(0,9)$ \\
\hline \multicolumn{12}{|c|}{$\begin{array}{l}\text { EP=Erro padrão } \\
\text { "Estimativa baseada em análise de regressão linear e ajustada para diferenças iniciais entre os grupos controle e intervenção quanto a idade, concentração de } \\
\text { hemoglobina, log de renda familiar per capita e duração do seguimento. } \\
{ }^{\dagger} p<0,000 \text { comparado com o ganho de hemoglobina do grupo controle. } \\
{ }^{5} \mathrm{p}<0,000 \text { para hipótese nula (nenhum ganho de } \mathrm{Hb} \text { ). }\end{array}$} \\
\hline
\end{tabular}


Tabela 3 - Distribuição das crianças dos grupos controle e intervenção de acordo com idade no inicio do seguimento.

Table 3 - Distribution of the studied children according to initial age and treatment group.

\begin{tabular}{cccc}
\hline \multirow{2}{*}{$\begin{array}{c}\text { Idade inicial } \\
\text { (meses) }\end{array}$} & Grupo controle & \multicolumn{2}{c}{ Grupo intervenção* } \\
\cline { 3 - 4 } & & Baixa adesão & Alta adesão \\
\hline $6-17$ & $53(18,1 \%)$ & $33(25,2 \%)$ & $39(18,1 \%)$ \\
$18-59$ & $240(81,9 \%)$ & $98(74,8 \%)$ & $176(81,9 \%)$ \\
$6-59$ & $293(100,0 \%)$ & $131(100,0 \%)$ & $215(100,0 \%)$ \\
\hline
\end{tabular}

* Baixa e alta adesão correspondem, respectivamente, à ingestão de menos do que $75 \%$ ou pelo menos $75 \%$ das doses do suplemento previstas ao longo do seguimento.

* Children with low compliance received less than $75 \%$, and those with high compliance received at least $75 \%$ of the recommended doses of supplement.

ança e grau de adesão, foram ajustadas por um modelo de regressão linear que leva em consideração diferenças entre os grupos controle e intervenção quanto à concentração inicial de hemoglobina, idade, logaritmo da renda familiar e duração do seguimento. Observa-se que o ganho na concentração de hemoglobina devido à intervenção (ganho líquido) foi bastante maior para as crianças com alto nível de adesão (5,0 contra 2,3 g/l no grupo de baixa adesão), particularmente no caso das crianças mais novas ( 6,6 contra $1,2 \mathrm{~g} / \mathrm{l})$. Ainda assim, impactos positivos da intervenção foram observados, em todas as idades, para crianças com baixa e alta adesão. Apenas entre crianças jovens com baixa adesão o impacto positivo da intervenção não alcançou significado estatístico.

A Tabela 5 apresenta os resultados da comparação entre os grupos controle e in- tervenção quanto à incidência de aumento ou diminuição relevantes na concentração de hemoglobina (equivalentes, respectivamente, a variações superiores $\mathrm{a}+10,0 \mathrm{~g} / \mathrm{l}$ ou inferiores a $-10,0 \mathrm{~g} / \mathrm{l})$. As razões de chance para aumento ou diminuição da concentração de hemoglobina - obtidas a partir de regressão logística, que também controla as diferenças iniciais entre dois grupos quanto à concentração de hemoglobina, idade, renda e duração do seguimento - indicam novamente vantagem para o grupo intervenção. Também novamente, os benefícios associados com a intervenção mostram-se diretamente relacionados ao grau de adesão dos beneficiários. Em geral, a intervenção parece funcionar melhor para prevenir reduções da concentração de hemoglobina do que para promover aumentos. Esse efeito preventivo da intervenção é particularmente notado entre as crianças que iniciaram a

Tabela 4 - Ganho ajustado líquido na concentração de hemoglobina ( $\mathrm{g} / \mathrm{l})$ devido à intervenção conforme idade inicial e nível de adesão.

Table 4 - Adjusted net gain in hemoglobin concentration ( $\mathrm{g} / \mathrm{l})$ due to intervention according to child's initial age and level of compliance.

\begin{tabular}{ccccccc}
\hline \multirow{2}{*}{$\begin{array}{c}\text { Idade inicial } \\
\text { (meses) }\end{array}$} & \multicolumn{2}{c}{ Total de crianças } & \multicolumn{4}{c}{ Grupo intervenção } \\
\cline { 3 - 7 } & & & \multicolumn{3}{c}{ Baixa adesão } & \multicolumn{2}{c}{ Alta adesão } \\
\hline & $-\mathrm{x} \pm \mathrm{EP}$ & $\mathrm{p}$ & $-\mathrm{x} \pm \mathrm{EP}$ & $\mathrm{p}$ & $-\mathrm{x} \pm \mathrm{EP}$ & $\mathrm{p}$ \\
$6-17$ & $4,1 \pm 2,7$ & 0,13 & $1,2 \pm 3,1$ & 0,69 & $6,6 \pm 3,0$ & 0,03 \\
$18-59$ & $4,0 \pm 0,9$ & $<0,01$ & $2,9 \pm 1,3$ & 0,03 & $4,7 \pm 1,0$ & $<0,01$ \\
$6-59$ & $4,0 \pm 0,9$ & $<0,01$ & $2,3 \pm 1,2$ & 0,06 & $5,0 \pm 1,0$ & $<0,01$ \\
\hline
\end{tabular}

$\mathrm{EP}=$ Erro Padrão/Standard Error

$\mathrm{p}=$ Probabilidade de incrementos negativos ou nulos na concentração de hemoglobina./probability of negative or null increases in hemoglobin concentration 
Tabela 5 - Razão de chances ajustada (intervalo de confiança de 95\% entre parênteses) para aumento (> $10 \mathrm{~g} / \mathrm{l}$ ) ou declínio $(<-10 \mathrm{~g} / \mathrm{l})$ da concentração de hemoglobina conforme idade inicial e grau de adesão à intervenção*.

Table 5 - Adjusted odds ratios (95\% confidence intervals in parentheses) for follow-up increasing (>+10.0 g/l) or decreasing (<$10.0 \mathrm{~g} / \mathrm{l})$ hemoglobin concentration according to the child's initial age and level of compliance*.

\begin{tabular}{|c|c|c|c|c|c|c|c|c|}
\hline \multirow{3}{*}{$\begin{array}{l}\text { Idade } \\
\text { inicial } \\
\text { (meses) }\end{array}$} & \multicolumn{4}{|c|}{ Aumento na concentração de hemoglobina } & \multicolumn{4}{|c|}{ Declínio na concentração de hemoglobina } \\
\hline & \multirow[t]{2}{*}{ Controle } & \multicolumn{3}{|c|}{ Intervenção } & \multirow[t]{2}{*}{ Controle } & \multicolumn{3}{|c|}{ Intervenção } \\
\hline & & $\begin{array}{l}\text { Baixa } \\
\text { adesão }\end{array}$ & $\begin{array}{c}\text { Alta } \\
\text { adesão }\end{array}$ & Total & & $\begin{array}{l}\text { Baixa } \\
\text { adesão }\end{array}$ & $\begin{array}{c}\text { Alta } \\
\text { adesão }\end{array}$ & Total \\
\hline $6-17$ & 1,0 & $\begin{array}{c}0,39 \\
(0,11-1,39)\end{array}$ & $\begin{array}{c}2,13 \\
(0,76-5,96)\end{array}$ & $\begin{array}{c}1,05 \\
(0,42-2,64)\end{array}$ & 1,0 & $\begin{array}{c}0,30 \\
(0,08-1,10)\end{array}$ & $\begin{array}{c}0,07 \\
(0,01-0,63)\end{array}$ & $\begin{array}{c}0,18 \\
(0,05-0,63)\end{array}$ \\
\hline $18-59$ & 1,0 & $\begin{array}{c}1,14 \\
(0,61-2,14)\end{array}$ & $\begin{array}{c}2,20 \\
(1,30-3,72)\end{array}$ & $\begin{array}{c}1,74 \\
(1,10-2,76)\end{array}$ & 1,0 & $\begin{array}{c}0,57 \\
(0,2-1,57)\end{array}$ & $\begin{array}{c}0,44 \\
(0,18-1,06)\end{array}$ & $\begin{array}{c}0,50 \\
(0,23-1,05)\end{array}$ \\
\hline $6-59$ & 1,0 & $\begin{array}{c}0,86 \\
(0,48-1,54)\end{array}$ & $\begin{array}{c}2,19 \\
(1,39-3,45)\end{array}$ & $\begin{array}{c}1,56 \\
(1,04-2,33)\end{array}$ & 1,0 & $\begin{array}{c}0,46 \\
(0,20-1,06)\end{array}$ & $\begin{array}{c}0,33 \\
(0,15-0,70)\end{array}$ & $\begin{array}{c}0,39 \\
(0,20-0,73)\end{array}$ \\
\hline
\end{tabular}

* Razões de chance ajustadas para idade inicial, concentração de hemoglobina inicial, log de renda familiar per capita e duração do seguimento.

* Odds ratios were adjusted for initial age, initial hemoglobin concentration, log of per capita family income, and duration of follow-up. Children with low compliance received less than $75 \%$, and those with high compliance received at least $75 \%$, of the recommended doses of the supplement.

intervenção com idades entre e 6 a 17 meses, faixa etária em que o risco de anemia é maior e as conseqüências são mais graves. Entre essas crianças, a intervenção reduziu em aproximadamente cinco vezes a chance de reduções relevantes na concentração de hemoglobina (razão de chances de 0,18), sendo que a redução foi ainda maior, 15 vezes, entre as crianças jovens que apresentaram alto grau de adesão à intervenção (razão de chances de 0,07).
A Tabela 6 apresenta predições relativas à prevalência da anemia infantil ao final do estudo nos grupos controle e intervenção. Essas predições foram obtidas modelandose a probabilidade individual de uma criança ser anêmica em função de sua participação na intervenção (controle ou beneficiário). Nas predições, a duração do seguimento foi fixada em sete meses (duração média do seguimento para o conjunto das crianças estudadas) e a concentração inicial

Tabela 6 - Prevalência estimada (intervalo de confiança de 95\% entre parênteses) de anemia (hemoglobina $<110 \mathrm{~g} / \mathrm{l}$ ) ao final do seguimento de acordo com idade inicial e grupo de tratamento*.

Table 6 - Predicted percent prevalence (95\% confidence intervals in parentheses) of anemia (hemoglobin < $110 \mathrm{~g} / \mathrm{l}$ ) at the end of follow-up according to the child's initial age and treatment group*.

\begin{tabular}{ccccc}
\hline \multirow{2}{*}{$\begin{array}{c}\text { Idade inicial } \\
\text { (meses) }\end{array}$} & Grupo & \multicolumn{3}{c}{ Grupo intervenção } \\
\cline { 3 - 5 } & controle & Baixa adesão & Alta adesão & Total de crianças \\
\hline $6-17$ & 58,2 & 55,8 & 24,5 & 38,5 \\
& $(41,9-72,9)$ & $(33,4-76,1)$ & $(13,7-40,0)$ & $(25,8-53,0)$ \\
$18-59$ & 13,6 & 6,7 & 4,2 & 5,0 \\
& $(9,4-9,3)$ & $(3,1-3,8)$ & $(2,5-7,2)$ & $(3,1-8,0)$ \\
$6-59$ & 21,6 & 14,1 & 6,4 & 9,1 \\
& $(16,3-27,9)$ & $(8,3-23,0)$ & $(4,1-9,9)$ & $(6,3-13,0)$ \\
\hline
\end{tabular}

"A duração do seguimento foi fixada em 7,0 meses e a concentração de hemoglobina inicial, idade inicial e a renda familiar per capita foram fixadas nos dois grupos com base nos valores médios encontrados na amostra de crianças entre 6 e 59 meses de idade da população da cidade de São Paulo.

"The duration of follow-up was set to 7.0 months, and the initial hemoglobin concentration, initial age, and logarithm of family income in each group were set to the average values found in the random sample of the child population of the city of São Paulo. 
de hemoglobina, a idade da criança na primeira visita e a renda familiar foram fixadas segundo os valores médios estimados a partir da amostra integral das crianças estudadas pelo inquérito realizado na cidade de São Paulo em 1995/96. Nessa medida, as prevalências que aparecem na tabela podem ser entendidas como uma previsão aproximada de qual seria a magnitude da anemia na cidade de São Paulo se a prescrição universal semanal de sulfato ferroso na infância tivesse ou não sido implementada em todo município.

Uma redução relativa de pouco mais de $50 \%$ na prevalência de anemia infantil poderia ser esperada na cidade de São Paulo caso a intervenção sob estudo fosse estendida para o conjunto da população entre 6 e 59 meses de idade (de 21,6\% para 9,1\%). Um impacto ainda maior (de $21,6 \%$ para $6,4 \%$, ou uma redução relativa de $70 \%$ ) poderia ocorrer caso se conseguisse para todas as crianças da cidade o nível de adesão observado no grupo de alta adesão (ingestão do suplemento em pelo menos $75 \%$ das semanas). As crianças maiores parecem responder melhor à intervenção, provavelmente porque a anemia mais severa (concentração de hemoglobina bem abaixo de $110 \mathrm{~g} / \mathrm{l}$ ) é mais comum nas crianças mais novas. A redução relativa na prevalência de anemia foi de $60 \%$ (de 13,6\% para 5,0\%) nas crianças maiores e 33\% (de $58,2 \%$ para $24,5 \%$ ) entre as crianças mais jovens. Se fosse possível maximizar a adesão à intervenção, a prevalência de anemia poderia ser reduzida em $70 \%$ entre as crianças maiores e em $57 \%$ nas crianças menores.

\section{Discussão}

Por questões éticas, os pais das crianças anêmicas do grupo controle foram informados do diagnóstico da enfermidade e aconselhados a buscar tratamento no serviço de saúde a que tivessem acesso. Esse procedimento ético, obrigatório, nem sempre tem sido observado em estudos que avaliam o impacto de intervenções que buscam o controle da anemia. Devido ao procedimento que adotamos, cerca de metade das crian- ças do grupo controle receberam algum tipo de suplemento durante o período de seguimento e, por essa razão, foram excluídas da análise final. As variações na hemoglobina desse grupo (não apresentadas nesse artigo) foram intermediárias entre os grupos controle e intervenção. Para lidar adequadamente com as diferenças entre os dois grupos, todas as comparações foram ajustadas pelos valores iniciais de hemoglobina e idade, além da renda familiar e do período de tempo entre as duas visitas.

$\mathrm{O}$ aumento líquido na concentração de hemoglobina atribuído à intervenção foi estimado em 4,0 g/l. Observou-se também que, entre as crianças que iniciaram a intervenção com 6 a 17 meses de idade, o risco de declínio na concentração de hemoglobina superior a $10 \mathrm{~g} / \mathrm{l}$ foi cinco vezes menor do que no grupo controle. Estendidos ao total de crianças do município de São Paulo, os resultados apontam uma redução superior a 50\% na prevalência de anemia. No grupo intervenção, quase dois terços das crianças receberam de seus pais pelo menos $75 \%$ das doses do suplemento semanal de ferro prescritas para o período. Conforme o esperado, o impacto da intervenção nesse grupo de crianças foi superior à média, mas mesmo no terço restante das crianças o impacto da intervenção foi positivo.

Conforme mencionado anteriormente, três ensaios realizados em populações asiáticas de pré-escolares demonstraram que a suplementação intermitente supervisionada de sais de ferro tem a mesma eficiência que a suplementação diária também supervisionada ${ }^{9-11}$. Uma meta-análise de 21 estudos que procuraram comparar a eficácia dos esquemas diário e intermitente de suplementação estimou que indivíduos em diferentes idades e grupos fisiológicos (crianças, adolescentes e gestantes) que receberam suplementos semanais de ferro tiveram uma prevalência final de anemia 1,34 vezes maior (intervalo 95\% confiança: 1,20 a 1,49) do que aqueles que recebiam a suplementação diária $^{23}$. A meta-análise em questão incluiu quatro estudos com crianças pré-escolares, dois estudos já publicados ${ }^{10,11}$ e dois ainda inédi- 
tos, todos conduzidos em populações préescolares asiáticas. Nesses quatro estudos, a prevalência final de anemia foi praticamente a mesma entre as crianças suplementadas semanal ou diariamente ${ }^{23}$. Entretanto, em apenas um desses estudos havia um grupo controle apropriado (crianças que não receberam suplemento ferroso), permitindo a estimativa da eficácia absoluta do esquema de suplementação semanal (comparação do esquema semanal com o grupo controle, em vez da comparação entre os esquemas semanal e diário). No referido estudo, a prevalência da anemia foi reduzida em cerca de $90 \%$, tanto com o esquema semanal quanto com o esquema diário de suplementação, enquanto entre as crianças do grupo controle a anemia aumentou cerca de $30 \%{ }^{11}$.

Conforme apontado pelos autores da meta-análise, a eficácia (capacidade de produzir efeitos desejados em condições experimentais) é requisito necessário mas não suficiente para determinar a efetividade de uma intervenção (capacidade de produzir os efeitos desejados sob condições usuais de operação). Uma vez que todos os estudos com pré-escolares ocorreram em condições muito controladas, os autores da meta-análise afirmam que seus resultados não podem assegurar que qualquer um dos métodos (diário ou semanal) será eficaz para controlar a deficiência de ferro e a anemia em programas regulares ${ }^{23}$.

O estudo realizado em São Paulo não foi delineado para avaliar a eficácia da suplementação semanal com ferro no tratamento de casos de anemia infantil, mas sim para explorar a efetividade no controle da anemia infantil de se recomendar, rotineiramente, a todas as crianças entre 6 e 59 meses de idade, a suplementação semanal com sais de ferro. Nesse sentido, deve-se notar que: 1) a ingestão do suplemento pela criança não foi supervisionada - os pais foram apenas aconselhados a fornecer o xarope a seus filhos; 2) as crianças que apresentaram baixa adesão à intervenção não foram excluídas da avaliação de impacto; e 3) os grupos controle e intervenção foram extraídos de uma amostra probabilística representativa da população infantil de uma localidade onde a prevalência da anemia alcança cerca de metade das crianças.

O fato de a recomendação para se ofertar semanalmente o suplemento ter sido realizada no contexto de um projeto de pesquisa, e não por pessoal regular de uma instituição de saúde, poderia ser considerado um argumento contrário à generalização do impacto observado. Porém é importante considerar que a equipe de pesquisa teve somente um único contato com as famílias ao início do estudo e que não mais do que cinco minutos foram gastos para explicar aos pais a necessidade de proteger suas crianças da anemia e a importância de ofertar o medicamento regularmente.

$\mathrm{O}$ fato de a prevalência final de anemia predita para o grupo de crianças entre 6 e 17 meses ter se apresentado relativamente alta (37,6\% comparado com prevalência de apenas $5,4 \%$ para crianças maiores) merece comentários adicionais. Poderiam ser cogitados dois possíveis motivos para essa elevada prevalência "residual" de anemia. O primeiro deles é referente ao menor grau de adesão à intervenção observado entre as crianças mais jovens: apenas $54 \%$ dessas crianças receberam pelo menos três quartos do total de doses prescritas do suplemento, comparadas com $64 \%$ entre as crianças mais velhas. No entanto, isso é seguramente uma explicação parcial, desde que a prevalência final foi ainda muito alta (24,5\%), mesmo quando apenas as crianças mais jovens com alta adesão foram consideradas. O segundo motivo, e provavelmente mais importante, é que o grupo de crianças mais jovens iniciou a intervenção, não apenas mais afetado pela anemia (prevalência inicial de anemia de $68,2 \%$ contra $35,2 \%$ entre as crianças mais velhas), como também com uma maior proporção de crianças com concentrações muito baixas de hemoglobina. Um terço das crianças anêmicas entre 6 e 17 meses de idade tinha concentrações de hemoglobina menores do que 9,0 g/l, contra um sexto entre as crianças mais velhas. É importante também considerar que, na população estudada, a 
prevalência de anemia severa (concentração de hemoglobina menor do que 9,0 g/l) aumenta rapidamente até os 18 meses de idade e decresce a partir dessa idade: $6,1 \%$ nos primeiros 6 meses de vida; $19,7 \%$ dos 6 aos 12 meses; $23,8 \%$ dos 12 aos 18 meses; $17,8 \%$ dos 18 aos 24 meses; $10,0 \%$ dos 24 aos 36 meses; 2,7\% dos 48 aos 60 meses (dados do estudo transversal). Isso indica que, para controlar eficazmente a anemia na faixa etária entre 6 e 17 meses, talvez seja necessário iniciar a suplementação semanal de ferro antes mesmo dos seis meses de idade.

A conclusão principal do presente estudo é de que a suplementação preventiva com doses semanais de ferro em crianças préescolares pode reduzir significativamente $o$ risco de anemia em condições não muito distantes daquelas que poderiam ser reproduzidas por serviços regulares de saúde pública. Essa conclusão é corroborada por um estudo de nove semanas conduzido em uma pequena cidade ao Leste da Ilha de Java, não incluído na meta-análise citada anteriormente. O referido estudo demonstrou que suplementos de ferro ofertados semanalmente pelas mães "nas condições normais de vida da comunidade" reduziram pela metade a prevalência de anemia entre crianças de dois a cinco anos de idade ${ }^{24}$.

\section{Referências}

1. DeMayer E, Dallman P, Gurney JM, Hallberg L, Sood SK, Srikantia SG. Preventing and controlling iron deficiency anaemia through primary health care: a guide for health administrators and programme managers. World Health Organization: Geneva; 1989.

2. Dallman PR, Siimes MA, Stekel A. Iron deficiency in infancy and childhood. Am J Clin Nutr 1980; 33: 86118.

3. Soemantri AG, Pollitt E, Kim I. Iron deficiency anaemia and educational achievement. Am J Clin Nutr 1985; 42: 1221-8.

4. Aukett MA, Parks YA, Scott PH, Wharton BA. Treatment with iron increases weight gain and psychomotor development. Arch Dis Childh 1986; 61: 849-57.

5. Lozzoff B, Jimenez E, Wolf W. Long-term developmental outcome of infants with iron deficiency. N Engl J Med 1991; 325: 687-93.

6. ACC/SCN. Second report on the world nutrition situation. Vol. 1. Global and regional results. ACC/ SCN: Geneva; 1992.

7. Monteiro CA, Szarfarc SC, Mondini L. Tendência secular da anemia na infância na cidade de São Paulo (1984-1996). Rev Saúde Pública 2000; 34 Suppl 6: 6272 .

8. ACC/SCN. Controlling iron deficiency. ACC/SCN state-of-the-art series. Geneva: ACC/SCN; 1991. (Nutrition policy discussion paper no 9 ).
9. Liu X-N, Kang J, Zhao L, Viteri FE. Intermittent iron supplementation in Chinese pre-school children is efficient and safe. Food Nutr Bull 1995; 16: 139-46.

10. Schultink W, Gross R, Gliwitzki M, Karyadi D, Matulessi P. Effect of daily vs twice weekly iron supplementation in Indonesian preschool children with low iron status. Am J Clin Nutr 1995; 61:11-5.

11. Thu B, Schultink W, Dillon D, Gross R, Leswara ND, Khoi HH. Effect of daily and weekly nutrient supplementation on micronutrient deficiencies and growth in young Vietnamese children. Am J Clin Nutr 1999; 69: 80-6.

12. Herbert V. Recommended dietary intakes (RDI) of iron in humans. Am J Clin Nutr 1987; 45: 679-86.

13. van Schenck H, Falkensson M, Lundberg B. Evaluation of "HemoCue", a new device for determining haemoglobin. Clin Chem 1986; 32: 526-9.

14. Hoffmann UA, Pons JA, Janer JL. The sedimentation concentration method in Schistossomiase mansoni, Puerto Rico. J Public Health 1934; 9: 283-91.

15. Thompson FE, Byers T. Dietary assessment resource manual. J Nutr 1994; 124: S 245-317.

16. Philippi ST, Szarfarc SC, Latterza AR. Virtual Nutri (software) - version 1.0 for Windows. São Paulo: Departamento de Nutrição da Faculdade de Saúde Pública da Universidade de São Paulo; 1996. 
17. FAO/WHO/UNU. Report of a Joint Expert Consultation. Energy and protein requirements. Geneva; 1985. (WHO - Technical Report Series, 724).

18. National Research Council. Recommended dietary allowances. $10^{\text {th }}$ ed. Washington, DC: National Academy Press; 1989.

19. Kirkwood BR. Essentials of medical statistics. London: Blackwell Scientific Publications; 1988.

20. Stata Corp. Stata Statistical Software: release 5.0. College Station, Texas: Stata Corporation; 1997.

21. Dean AG, Dean JA, Burton AH, Dicker RC. Epi Info, version 6 bc: a word processing, database, and statistics program for epidemiology on microcomputers. Georgia: Center for Disease Control and Prevention; 1997.
22. Hamill PVV, Dirzd TA, Johnson CL, Reed RB, Roche AF, Moore WM. Physical growth: NCHS percentiles. Am J Clin Nutr 1979; 32: 607-29.

23. Beaton GH, McCabe GP. Efficacy of intermittent iron supplementation in the control of iron deficiency anaemia in developing countries: an analysis of experience. Final report to the micronutrient initiative. Toronto, Canada: GHB Consulting; 1999.

24. Palupi L, Schultink W, Achadi E, Gross R. Effective community intervention to improve haemoglobin status in preschoolers receiving once-weekly iron supplementation. Am J Clin Nutr 1997; 65: 1057-61.

Recebido em 04/01/02; aprovado em 15/08/02 